Orinoquia, Julio-Diciembre 2019;23(2):79-86

ISSN electrónico 2011-2629.

ISSN impreso 0121-3709.

https://doi.org/10.22579/20112629.571

\title{
Crecimiento poblacional de Macrothrix spinosa alimentada con Chlorella sp.
}

\author{
Population growth of Macrothrix spinosa fed with Chlorella sp.
}
Crescimento populacional de Macrothrix spinosa alimentado com Chlorella sp.

\author{
Harold DJ Oviedo-Montiel'; ${ }^{1}$ Edwin E. Herrera-Cruz ${ }^{2}$; Jenny K. Hoya-Florez ${ }^{3}$; \\ Martha J Prieto-Guevara ${ }^{4}$; Ana L. Estrada-Posada ${ }^{5}$ Jonny A. Yepes-Blandón ${ }^{6}$
}

1 Prof Acuiclt, (c)MSc, Grupo de Investigación en Peces Nativos-GIPEN, Piscícola San Silvestre S.A., Barrancabermeja Santander

2 Zoot, (c)MSc, Grupo de Investigación en Peces Nativos-GIPEN, Piscícola San Silvestre S.A., Barrancabermeja Santander 3 MVZ, (c)MSc, Grupo de Investigación en Peces Nativos-GIPEN, Piscícola San Silvestre S.A., Barrancabermeja Santander

4 Biol, Esp, MSc, PhD, Universidad de Córdoba, Facultad de Medicina Veterinaria y Zootecnia, Departamento de Ciencias Acuícolas, Instituto de Investigaciones Piscícolas

5 Biol, MSc, (c)PhD, ISAGEN S.A

6 Zoot, MSc, (c)PhD, Grupo de Investigación en Peces Nativos-GIPEN, Piscícola San Silvestre S.A., Barrancabermeja Santander

Email: investigacionpisansilvestre@gmail.com

\section{Resumen}

El zooplancton es considerado como un alimento de gran importancia para las larvas de los peces por su excelente perfil nutricional. La mayor dificultad en el cultivo del zooplancton, en especial de cladóceros, es la susceptibilidad al alimento que consumen, pues deficiencias en los nutrientes influyen significativamente en su producción. Por tanto, el estudio de la partícula alimenticia en cantidad y calidad óptima es necesario para potencializar la producción. En la Piscícola San Silvestre S.A. se evaluó el efecto del alimento sobre las variables productivas del cladócero Macrothrix spinosa con fotoperiodo 12:12 luz: oscuridad y aireación constante en 8 unidades experimentales con volumen de 2,5 L. Los organismos, en densidad inicial de $2 \mathrm{org} / \mathrm{mL}$, se alimentaron con la microalga Chlorella sp, previamente cultivada en dos medios de cultivo: (T1) Chlorella sp. cultivada con F/2 de Guillard y (T2) Chlorella sp. cultivada con Nutrifoliar $§$. Fueron determinados los parámetros poblacionales: densidad máxima (Dm), tasa instantánea de crecimiento (K), tiempo de duplicación (Td) y rendimiento $(\mathrm{R})$. Diariamente se registró la temperatura $\left(25,86 \pm 0,36^{\circ} \mathrm{C}\right), \mathrm{pH}(7,58 \pm 0,32)$ y $\mathrm{OD}(5,74 \pm 0,56 \mathrm{mg} / \mathrm{L})$. La mayor Dm fue 27,38 $\pm 0,08 \mathrm{org} / \mathrm{mL}$ en T1 (P>0,05). Mayor K, menor Td y mayor R se registraron en T1 $(0,24 \pm 0,00,2,84 \pm 0,04$ días y $2,50 \pm 0,01 \mathrm{org} / \mathrm{mL}$ respectivamente) $(\mathrm{P}>0,05)$. Los resultados sugieren que $M$. spinosa, alimentada con la microalga Chlorella sp. cultivada con F/2 de Guillard, alcanza mejor desempeño poblacional en cultivo.

Palabras claves: Alimento vivo; Microalgas; Cladócero; F/2 de Guillard; Nutrifoliar 


\begin{abstract}
Zooplankton is considered a food of great importance for fish larvae because of its excellent nutritional profile. The greatest difficulty in the culture of zooplankton, especially cladocerans, is the susceptibility to the food they consume, since deficiencies in the nutrients significantly influence their production. Therefore, the study of the food particle in optimal quantity and quality is necessary to potentiate production. In the Piscícola San Silvestre S.A, the effect of the food on the productive variables of the cladoceran Macrothrix spinosa with photoperiod 12:12 light: dark and constant aeration in 8 experimental units with volume of 2,5 liters was evaluated. The organisms, in initial density of $2 \mathrm{clad} / \mathrm{mL}$, were fed with the microalga Chlorella sp, previously cultivated in two culture media: (T1) Chlorella sp. cultivated with Guillard's F/2 and (T2) Chlorella sp. grown with Nutrifoliar ${ }^{\circledR}$. The population parameters were determined: maximum density $(\mathrm{Dm})$, instantaneous growth rate $(\mathrm{K})$, doubling time $(\mathrm{Td})$ and yield $(\mathrm{R})$. The temperature was recorded daily $\left(25,86 \pm 0,36{ }^{\circ} \mathrm{C}\right), \mathrm{pH}(7,58 \pm 0,32)$ and $\mathrm{OD}$ $(5,74 \pm 0,56 \mathrm{mg} / \mathrm{L})$. The highest Dm was $27,38 \pm 0,08 \mathrm{org} / \mathrm{mL}$ in $\mathrm{T} 1(\mathrm{P}>0.05)$. Higher $\mathrm{K}$, lower Td and higher $\mathrm{R}$ were recorded in $\mathrm{T} 1(0,24 \pm 0,00,2,84 \pm 0,04$ days and $2,50 \pm 0,01 \mathrm{org} / \mathrm{mL}$ respectively) $(\mathrm{P}>0,05)$. The results suggest that $\mathrm{M}$. spinosa fed with the microalga Chlorella sp. cultivated with Guillard's F / 2, achieves better population performance in culture.
\end{abstract}

Key words: Live food; Microalgae; Cladocera; Guillard's F/2; Nutrifoliar

\begin{abstract}
Resumo
O zooplâncton é considerado um alimento de grande importância para as larvas de peixes, devido ao seu excelente perfil nutricional. A maior dificultade na cultura do zooplâncton, especialmente dos cladóceros, é a suscetibilidade aos alimentos que consomem; uma vez que deficiências nos nutrientes influenciam significativamente sua produção. Portanto, o estudo da partícula alimentar em quantidade e qualidade ótimas é necessário para potencializar a produção. Na Piscícola San Silvestre S.A, foi avaliado o efeito do alimento sobre as variáveis produtivas do cladoceran Macrothrix spinosa com fotoperíodo 12:12 luz: escura e aeração constante em 8 unidades experimentais com volume de 2,5 litros. Os organismos em densidade inicial de $2 \mathrm{org} / \mathrm{mL}$ foram alimentados com a microalga Chlorella sp., previamente cultivada com dois meios de cultura: (T1) Chlorella sp. cultivada com F/2 de Guillard e (T2) Chlorella sp. cultivada com Nutrifoliarß. Os parâmetros populacionais foram determinados: densidade máxima $(\mathrm{Dm})$, taxa de crescimento instantâneo $(\mathrm{K})$, tempo de duplicação $(\mathrm{Td})$ e rendimento $(\mathrm{R})$. A temperatura foi registrada diariamente $\left(25,86 \pm 0,36{ }^{\circ} \mathrm{C}\right), \mathrm{pH}(7,58 \pm 0,32)$ e $\mathrm{OD}(5,74 \pm 0,56 \mathrm{mg} / \mathrm{L})$. O maior Dm foi de 27,38 $\pm 0,08$ org/ $\mathrm{mL}$ em T1 (P> 0,05). Maior K, menor Td e maior R foram registrados em T1 $(0,24 \pm 0,00$, $2,84 \pm 0,04$ dias e $2,50 \pm 0,01 \mathrm{org} / \mathrm{mL}$, respectivamente) $(\mathrm{P}>0,05)$. Os resultados sugerem que $M$. spinosa alimentada com a microalga Chlorella sp. cultivada com F/2 de Guillard, alcança melhor desempenho populacional em cultura.
\end{abstract}

Palavras-chave: Alimento vivo; Microalgas; Cladocera; F/2 de Guillard; Nutrifoliar

\section{Introducción}

Dentro de la gran diversidad de zooplancton, los cladóceros son considerados como organismos de importancia en acuicultura por el rápido crecimiento poblacional, la variabilidad de tamaño en el desarrollo y por ser presas fáciles para capturar y digerir (SipaúbaTavares et al., 2014; Pérez et al., 2015; Luna-Figueroa y Arce, 2017). Además, la composición nutricional de estos organismos es esencial en la primera alimentación de peces; presentan proteína (45-78 \%), lípidos (15$40 \%$ ), ácidos grasos esenciales (AGE), aminoácidos (aa) y vitaminas, así como, tienen un amplio espectro de enzimas que contribuyen en el óptimo desarrollo de las larvas (Pérez et al., 2015; Luna-Figueroa y Arce, 2017; Khudyi et al., 2018; Cheban et al., 2018; Mellisa et al., 2018).

En cladóceros, la calidad y disponibilidad del agua y del alimento influyen en el crecimiento, la reproducción y la sobrevivencia (Ismail et al., 2011; Luna-Figueroa y Arce, 2017; Dutta et al., 2017; Wei et al., 2018). La variación o deficiencia en los nutrientes, interviene sobre la fecundidad y la longevidad, lo que condicio- na la dinámica poblacional (Pérez et al., 2015; LunaFigueroa y Arce, 2017; Kar et al., 2017). Este tipo de zooplancton por su capacidad filtradora, se alimenta de bacterias, materia orgánica y microalgas (SipaúbaTavares et al., 2014; Castro-Mejia et al., 2017; Marinho et al., 2017).

Las microalgas, son el ítem de mayor consumo, debido a su alto perfil nutricional, contienen la mayor proporción de macronutrientes y sustancias biosintetizadoras como la clorofila (Prieto-Guevara 2013; Muñoz et al., 2013; Sipaúba-Tavares et al., 2014). Sin embargo, la calidad nutricional de éstas depende de las condiciones de temperatura, $\mathrm{pH}$, luz y medio de cultivo (VásquezSuárez et al., 2013). Este último, influye sobre el crecimiento, la productividad y la concentración de los nutrientes en las microalgas (Silva-Benavides, 2016).

Por lo anterior, es importante la selección del sustrato que optimice la producción de la dieta de cladóceros y que, además, ofrezca los nutrientes necesarios para el desarrollo en cultivo y paralelamente potencialice su uso como presa viva. Por tanto, la presente investigación evaluó el efecto de la microalga Chlorella sp., 
cultivada en dos medios de cultivo, sobre el crecimiento poblacional y variables productivas del cladócero Macrothrix spinosa.

\section{Materiales y métodos}

El estudio se desarrolló en el Laboratorio de Alimento Vivo de la Piscícola San Silvestre (LAVPSS), Barrancabermeja, Colombia. El cladócero Macrothrix spinosa, fue aislado de los estanques de la piscícola y mediante la técnica de micropipeteo manual en laboratorio, bajo observación directa al estereoscopio (Leica EZ4 $W)$, en condiciones de temperatura $\left(24^{\circ} \mathrm{C}\right)$, fotoperiodo (10:14 horas luz: oscuridad), iluminación indirecta (lámparas led fluorescentes de 1350 lumens E-TLT818G13P 18W)) fue caracterizado e identificado de acuerdo a las claves taxonómicas de Fuentes-Reines et al., (2012). El mantenimiento de la cepa, se realizó en frascos de vidrio translucidos de $200 \mathrm{~mL}$, con agua filtrada y renovación del 30\% del volumen cada 72 horas y limpieza de fondo para mantener la calidad del agua, alimentada con la dieta mixta de las microalgas Chlorella sp., Desmodesmus sp. y Ankistrodesmus sp.

\section{Cultivo de Chlorella sp.}

La microalga Chlorella sp fue aislada de los estanques de la Piscícola San Silvestre S.A., identificada de acuerdo a las características reportadas por Guamán y González, (2016) y mantenida en el Laboratorio de Alimento Vivo (LAVPSS) bajo las normas mínimas de asepsia en laboratorio. La microalga se cultivó en un medio tradicional, F/2 de Guillard (Guillard y Ryther, 1962) que contiene macronutrientes como $\mathrm{NaNO}_{3}(75 \mathrm{~g} / \mathrm{L}), \mathrm{NaH}_{2} \mathrm{PO}_{4} \mathrm{H}_{2} \mathrm{O}(5 \mathrm{~g} / \mathrm{L})$, Metales traza, $\mathrm{CuSO}_{4} 5 \mathrm{H}_{2} \mathrm{O}(0,19 \mathrm{~g} / \mathrm{L}), \mathrm{ZnSO}_{4} 7 \mathrm{H}_{2} \mathrm{O}(1,0 \mathrm{~g} / \mathrm{L})$, Co$\mathrm{Cl}_{2} 6 \mathrm{H}_{2} \mathrm{O}(0,5 \mathrm{~g} / \mathrm{L}), \mathrm{MnCl}_{2} 4 \mathrm{H}_{2} \mathrm{O}(9,0 \mathrm{~g} / \mathrm{L}), \mathrm{Na}_{2} \mathrm{MoO}-$ ${ }_{4} 2 \mathrm{H}_{2} \mathrm{O}(0,31 \mathrm{~g} / \mathrm{L}), \mathrm{Na}_{2} \operatorname{EDTA}(4,36 \mathrm{~g} / \mathrm{L}), \mathrm{FeCl}_{3} 6 \mathrm{H}_{2} \mathrm{O}$ $(3,15 \mathrm{~g} / \mathrm{L})$ y vitaminas (Thiamine 0,1 mg, Biotin 0,1 g/L y Cyanocobalamina 0,5 g/L) y, un fertilizante agrícola de bajo costo, Nutrifoliar completo $\AA$, característico por contener elementos mayores como nitrógeno total $(200 \mathrm{~g} / \mathrm{L}), \mathrm{P}_{2} \mathrm{O}_{3}(100 \mathrm{~g} / \mathrm{L}), \mathrm{K}_{2} \mathrm{O}(50 \mathrm{~g} / \mathrm{L})$; secundarios como $\mathrm{MgO}(10 \mathrm{~g} / \mathrm{L}), \mathrm{S}(14 \mathrm{~g} / \mathrm{L})$ y micronutrientes como B (1,5 g/L), Cu $(2,5 \mathrm{~g} / \mathrm{L})$, Fe $(1,0 \mathrm{~g} / \mathrm{L})$, Mn $(1,0$ $\mathrm{g} / \mathrm{L})$, Mo $(0,03 \mathrm{~g} / \mathrm{L})$ y Zn $(5,0 \mathrm{~g} / \mathrm{L})$. El fertilizante se preparó a razón de $0.9 \mathrm{~mL} / \mathrm{L}$ de agua a nutrir.

El sistema de producción se realizó en lotes o "batch", en condiciones asépticas con inoculo de material biológico desde tubos de ensayo $(10 \mathrm{~mL})$ en medio líquido, escalonadamente hasta volúmenes de $250 \mathrm{~mL}$, 1 y 3 litros en frascos de vidrio. La disponibilidad de microalgas para la alimentación de los cladóceros, se mantuvo constante mediante cultivo monoalgal con temperatura $\left(24^{\circ} \mathrm{C}\right)$ y luz constante $(24$ horas/día con lámparas led fluorescentes de 1350 lumens (E-TLT818G13P 18W) y la aireación mediante uso de manguera plástica $(5.0 \mathrm{~mm}$ de diámetro) conectada a un sistema de suministro de aire con Blowers de $1.5 \mathrm{Hp}$.

\section{Cultivo de M. spinosa}

Los cladóceros se cultivaron en unidades experimentales de vidrio traslúcido con volumen útil de 2,5 L con inóculos de $2 \mathrm{org} / \mathrm{mL}$; aireación individual a través de aireador de 1HP, manguera plástica de 5,0 mm de diámetro con piedra difusora; fotoperiodo 10:14 horas (Luz: Oscuridad).

El efecto de la dieta sobre el crecimiento poblacional de M. spinosa, se evaluó durante 24 días con dos dietas microalgales en la alimentación de los organismos con cuatro réplicas por cada tratamiento: (T1) Chlorella sp. cultivada con F/2 de Guillard y (T2) Chlorella sp. cultivada con Nutrifoliar®. La concentración de las partículas alimenticias se mantuvo constante en $4 \times 10^{5}$ $\mathrm{cel} / \mathrm{mL}$, mediante recuento en cámara Neubauer (1/10 $\mathrm{mm}$ deep, Bright line-Boeco, Germany) y bajo microscopio óptico (Leica DM 500, USA) en cada unidad experimental cada 48 horas, previo a la alimentación y, se realizó reposición del alimento consumido.

Cada 48 horas se realizó recuento de organismos en cinco alícuotas de $1 \mathrm{~mL}$ por cada unidad experimental, bajo observación directa al estereoscopio (Leica EZ4 W). Los datos de densidad poblacional registrados durante el periodo experimental permitieron determinar las variables de crecimiento poblacional (González et al., 1988): tasa instantánea de crecimiento $(\mathrm{K})$, tiempo de duplicación $(\mathrm{Td})$, rendimiento $(\mathrm{R})$ y densidad máxima $(\mathrm{Dm})$.

Durante la fase experimental se registró diariamente: oxígeno disuelto (OD), temperatura $\left(\mathrm{T}^{\circ} \mathrm{C}\right)$ y $\mathrm{pH}$ con un YSI (Profesional Series 110, pH 100A, USA). Además, se realizó recambio cada 48 horas del 30 a $50 \%$ de agua del volumen total, que coincidía con la reposición del alimento, con el fin de mantener las condiciones óptimas para M. spinosa.

\section{Diseño experimental y análisis estadístico}

El análisis del crecimiento poblacional del cladócero Macrothrix spinosa, se realizó bajo un diseño completamente al azar. Los datos registrados se sometieron a pruebas de normalidad y homogeneidad de varianza. Los valores fueron expresados como media \pm desviación estándar. Análisis de ANOVA y prueba de Rangos Múltiples de Tukey o análisis no paramétrico de Dunett se realizaron 
para establecer diferencia estadística. En todos los casos fue utilizado un 95\% de confianza $(\mathrm{P}<0,05)$. El análisis estadístico se desarrolló con la ayuda del software IBM SPSS Statistics, versión 23.0.0.0, 2015.

\section{Resultados}

En cultivo, M. spinosa se caracterizó por presentar durante 24 días una curva poblacional diferente entre tratamientos (Figura 1). La fase de adaptación se presentó hasta el día dos sin variación en la densidad. La fase de crecimiento exponencial con producción acelerada de cladóceros se presentó entre los días tres y doce, con máxima densidad al día 12 para T1, y el día 10 para T2 con incremento moderado de densidad. Finalmente, se presentó la fase de decrecimiento exponencial a partir de los días 14 y 12 para T1 y T2 respectivamente hasta el día 24, cuando la tasa de mortalidad fue mayor a la tasa de fecundidad (Figura 1).

Diariamente a partir del día seis hasta el final del ciclo de cultivo se observó diferencia significativa en la densidad máxima alcanzada $(\mathrm{P}<0,05)$ entre los tratamientos (Figura 2). En T1 se registró 27,38 $\pm 0,08$ org/mL en el día 12, mientras que en T2 sólo se alcanzó el 50\% de T1 $(13,50 \pm 0,14 \mathrm{org} / \mathrm{mL})$ en el día 11 de cultivo.

En cuanto a los parámetros poblacionales evaluados, se encontró que el mayor valor de $\mathrm{K}(0,24 \pm 0,00)$, $R$ $(2,50 \pm 0,01 \mathrm{org} / \mathrm{mL}), \mathrm{Dm}(27,38 \pm 0,08 \mathrm{org} / \mathrm{mL})$ y el menor $\mathrm{Td}$ (6,94 \pm 0,11 días) se registró en T1 con diferencias estadísticas significativas entre tratamientos $(\mathrm{P}<0,05)$ para los cuatro parámetros (Tabla 1).

En relación con la matriz agua, la temperatura durante el cultivo de $M$. spinosa se registró sobre los $25^{\circ} \mathrm{C}$, el oxígeno superó los $5 \mathrm{mg} / \mathrm{L}$ y el pH fue neutro; no se encontraron diferencias significativas para las variables de calidad de agua entre los dos tratamientos $(P>0,05)$ (Tabla 2).

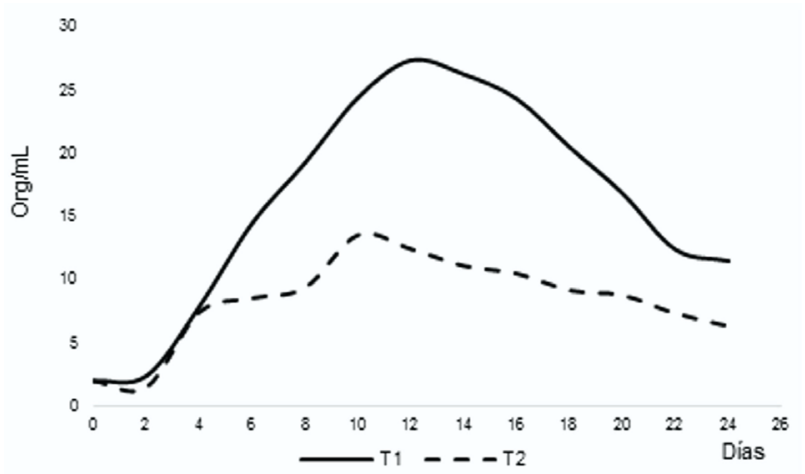

Figura 1. Curva de crecimiento poblacional de M. spinosa en 2,5L alimentada con Chlorella sp. cultivada con dos medios: F/2 de Guillard (T1) y Nutrifoliar@. (T2).

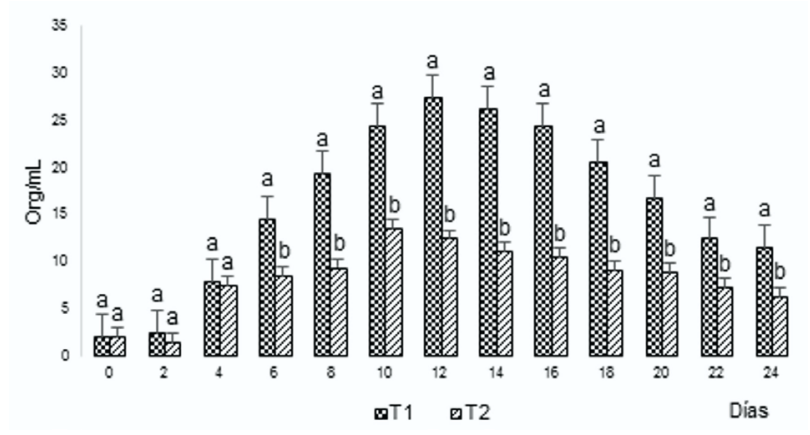

Figura 2. Densidad diaria de M. spinosa en cultivo de $2.5 \mathrm{~L}$ alimentada con Chlorella sp. cultivada con: F/2 de Guillard (T1) y Nutrifoliar ${ }^{\circledR}(\mathrm{T} 2)$. Letras diferentes en el mismo día expresan diferencia significativa $(P<0,05)$.

Tabla 1. Parámetros poblacionales (Promedio \pm DV) de $M$. spinosa evaluados en cultivo de 2,5 litros alimentada con Chlorella sp. cultivada con dos medios: F/2 de Guillard (T1) y Nutrifoliar $囚$. (T2). K: Tasa instantánea de crecimiento, Td: Tiempo de duplicación, R: Rendimiento, Dm: Densidad máxima.

\begin{tabular}{|l|c|c|}
\hline \multicolumn{1}{|c|}{ Variable } & T1 & T2 \\
\hline$K^{*}$ & $\mathbf{0 , 2 4} \pm \mathbf{0 , 0 0}^{\mathrm{a}}$ & $0,10 \pm 0,00^{\mathrm{b}}$ \\
\hline $\mathrm{Td}(\mathrm{días})^{* *}$ & $2,84 \pm 0,04^{\mathrm{a}}$ & $\mathbf{6 , 9 4} \pm \mathbf{0 , 1 1 ^ { b }}$ \\
\hline $\mathrm{R}(\mathrm{org} / \mathrm{mL})^{* *}$ & $\mathbf{2 , 5 0} \pm \mathbf{0 , 0 1 ^ { \mathrm { a } }}$ & $1,01 \pm 0,02^{\mathrm{a}}$ \\
\hline $\mathrm{Dm}(\mathrm{org} / \mathrm{mL})^{*}$ & $\mathbf{2 7 , 3 8} \pm \mathbf{0 , 0 8}^{\mathrm{a}}$ & $13,50 \pm 0,14^{\mathrm{b}}$ \\
\hline
\end{tabular}

Letras diferentes en las filas indican diferencia significativa $(\mathrm{P}<0.05)$. * Prueba de Tukey, ** Prueba de Dunett. Valores mayores en negrita.

\section{Discusión}

En esta investigación se observó incidencia de la naturaleza del alimento sobre el crecimiento y los parámetros reproductivos de $M$. spinosa. Las mejores variables productivas se presentaron cuando los organismos se alimentaron con Chlorella sp. cultivada con medio

Tabla 2. Parámetros de calidad del agua en el cultivo de $M$. spinosa en 2,5L alimentada con Chlorella sp. cultivada en dos medios: T1: F/2 de Guillard; T2: Nutrifoliar ${ }^{\circledR}$ (Promedio \pm DV).

\begin{tabular}{|l|c|c|}
\hline \multicolumn{1}{|c|}{ Variable } & T1 & T2 \\
\hline Temperatura $\left({ }^{\circ} \mathrm{C}\right)$ & $25,86 \pm 0,36^{\mathrm{a}}$ & $25,82 \pm 0,44^{\mathrm{a}}$ \\
\hline $\begin{array}{l}\text { Oxígeno disuelto } \\
\left(\mathrm{mg} \cdot \mathrm{L}^{-1}\right)\end{array}$ & $5,67 \pm 0,36^{\mathrm{a}}$ & $5,74 \pm 0,56^{\mathrm{a}}$ \\
\hline $\mathrm{pH}$ & $7,61 \pm 0,32^{\mathrm{a}}$ & $7,58 \pm 0,32^{\mathrm{a}}$ \\
\hline
\end{tabular}

Valores con letras diferentes en las filas indican diferencia estadística $(\mathrm{P}<0,05)$. 
F/2 de Guillard (T1). En contraste, menor eficiencia se presentó al alimentar con Chlorella sp. cultivada con Nutrifoliar® (T2). Esto puede estar relacionado con los requerimientos nutricionales fundamentales en la fase de reproducción de cladóceros, que se ven afectados cuando las condiciones alimenticias son desfavorables.

El valor nutricional de las microalgas ofrecidas como alimento para organismos acuáticos está determinado por el medio de cultivo empleado (Silva-Benavides, 2016). En esta investigación, se proporcionaron altas concentraciones de nitrógeno con el fertilizante Nutrifoliar®; al respecto, Cobos-Ruiz et al., (2016) aseguran que al aumentar el nitrógeno en el cultivo de Chlorella sp. se obtiene mayor contenido de proteínas y carbohidratos, pero menor concentración de lípidos y ácidos grasos.

En contexto, una disminución en la concentración de ácidos grasos en la dieta de cladóceros, ocasiona una deficiencia energética en el alimento, lo que inhibe la tasa de reproducción, aumenta el tiempo entre desoves y extiende el periodo de desarrollo embrionario, afectando la fecundidad y la regulación del crecimiento poblacional (Viti et al., 2013; Gándara et al., 2013; Silva et al., 2014; Sipaúba-Tavares et al., 2014; Luna-Figueroa et al., 2017). Por tanto, una dieta baja en ácidos grasos no cubre los gastos requeridos en la reproducción, el crecimiento y demás procesos metabólicos.

Sobre el tema, Fileto et al., (2007) e Ismail et al., (2011) aseveran que bajos niveles de ácidos grasos poliinsaturados en el alimento de estos organismos, generan bajo crecimiento en la población, esto atribuido a bajos niveles de energía por la partícula alimenticia. En esta investigación se puede relacionar el bajo rendimiento de M. spinosa alimentados con Chlorella sp. cultivada con Nutrifoliar ${ }^{\circ}$, a las concentraciones de nitrógeno que este fertilizante presenta, las cuales pueden disminuir el contenido de lípidos y ácidos grasos, fundamentales para los mecanismos reproductivos de cladóceros.

Otro aspecto fundamental en el cultivo de cladóceros, es la concentración del alimento suministrado. Un exceso en las concentraciones de la partícula alimenticia puede interferir en la longevidad de los organismos al afectar su proceso de filtración (Day et al., 2017), lo que puede ocasionar una disminución en el consumo, esto a su vez conlleva a una reducción en la calidad reproductiva. Castilho et al., (2012), señalan baja fecundidad en hembras del cladócero Scapholeberis armata, lento desarrollo y mayor edad de la primípara cuando se suministró una elevada concentración alimenticia.
En contraste, baja densidad del alimento disponible en cultivo, inhibe la fecundidad e interviene en la dinámica poblacional (Azuraidi et al., 2013; Sipaúba-Tavares et al., 2014).

En síntesis, Prieto-Guevara (2013) y Otero et al., (2013) manifiestan que una concentración de microalgas entre $3 \times 10^{5}$ a $4 \times 10^{5} \mathrm{cel} / \mathrm{mL}$ es recomendable para el mantenimiento y producción de diferentes especies de cladóceros. En esta investigación, la concentración de la partícula alimenticia se mantuvo en $4 \times 10^{5} \mathrm{cel} / \mathrm{mL}$ en ambos tratamientos, por tanto, se infiere que la variación en la dinámica poblacional en los tratamientos obedece al tipo de alimento y posiblemente a su valor nutricional y no a la concentración alimenticia.

Referente a la máxima densidad de organismos alcanzada, esta es condicionada por la temperatura, la densidad del inóculo, el tamaño del organismo, la concentración y la calidad del alimento, entre otros (Wang et al., 2009; Huang et al., 2011; Azuraidi et al., 2013; Chen et al., 2016). Generalmente, cuando la disponibilidad y calidad del alimento es adecuada, la población de cladóceros aumenta rápidamente, alcanzando altas densidades en cortos periodos de tiempo (Azuraidi et al., 2013). En esta investigación, la máxima densidad $(27,38 \pm 0,08 \mathrm{org} / \mathrm{mL})$ se presentó en T1 en tan solo 12 días, esta densidad puede considerarse como alta según el reporte de otros estudios. Los hallazgos de la presente investigación son similares a lo reportado por Azuraidi et al., (2013), en cuanto al día de máxima densidad. Los autores registraron la mayor densidad de Moina micrura (4,86 org/mL) para el día 10 de cultivo. No obstante, la densidad en esta investigación fue su-

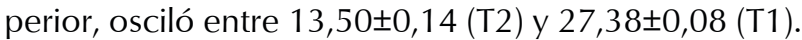
Resultados inferiores a esta investigación, los reportan Espinosa-Rodríguez et al., (2012), para la especie Macrothrix triserialis, alcanzando la máxima densidad (6.5 org/mL) en el día 23 de cultivo. Mientras Güntzel et al., (2003) realizaron una proyección de cultivo de la especie Macrothrix flabelligera, con base en la fecundidad, edad de la primípara y longevidad de la especie, calculando que se logra la máxima densidad entre los 10 y 15 días de cultivo, superando los 20 org/mL de M. flabelligera. Estos datos son similares a los obtenidos en esta investigación, por tanto, se deduce que $M$. spinosa logra adecuadas densidades en cultivo, siempre y cuando sea alimentada con una dieta óptima, en este caso con Chlorella sp. cultivada con F/2 de Guillard.

En contexto, durante el ciclo evaluado los mejores valores de las variables $\mathrm{K}, \mathrm{Td}$, y $\mathrm{R}$, se presentaron cuando M. spinosa se alimentó con Chlorella sp. cultivada con medio F/2 de Guillard (T1), duplicando la produc- 
tividad de T2. Como se mencionó anteriormente, la adecuada calidad del alimento suple las necesidades energéticas de estos organismos, favorece las tasas de reproducción y disminuye el tiempo de desarrollo embrionario, por consecuencia el tiempo entre desoves (Fileto et al., 2007; Azuraidi et al., 2013). Este efecto de la calidad del alimento se ve reflejado en el aumento de la población en cortos periodos de tiempo (Ismail et al., 2011; Sipaúba-Tavares et al., 2014). Por tanto, se puede establecer que los parámetros poblacionales de $M$. spinosa son condicionados por la calidad del alimento suministrado.

Los rangos de los parámetros poblacionales alcanzados por M. spinosa son similares a los reportados por diversos estudios para otros cladóceros de importancia acuícola. Azuraidi et al., (2013) reportaron para M. micrura una tasa de crecimiento de 0,22 $\pm 0,01$ a 0,31 00,01 días. A su vez, Otero et al., (2013), para Diaphanosoma sp. y Alona sp., registraron valores de $\mathrm{K}(0,13 \pm 0,07$ y $0,15 \pm 0,04$ días $), R(0,01 \pm 0,004$ y 0,019 $\pm 0,01 \mathrm{org} / \mathrm{mL} /$ día $)$ y $\mathrm{Td}(5,2 \pm 0,5$ y $4,3 \pm 0,38$ días) al alimentar con una dieta mixta de Chlorella vulgaris + levadura en concentración de $4 \times 10^{5}$ cel/ $\mathrm{mL}$. Prieto-Guevara (2013) alimentó con dieta mixta de Ankistrodesmus sp + Levadura $\left(40 \times 10^{4} \mathrm{cel} / \mathrm{mL}\right)$ al cladócero Moina sp., registrando valores superiores de $K(0,35 \pm 0,02), \operatorname{Td}(1,94 \pm 0,012)$, menores valores de $\mathrm{R}(1,1 \pm 0,02)$ y máxima densidad $(12,3 \pm 0,30 \mathrm{org} / \mathrm{mL})$. Resultados similares a Prieto-Guevara (2013), fueron reportados por Chen et al., (2016), que alcanzaron valores de $\mathrm{K}(0,6)$ cuando alimentó con una suspensión de Chlorella pyrenoidosa (2,0 mg/L).

Con base en lo anterior, se establece que $M$. spinosa alimentada con Chlorella sp. cultivada en F/2 de Guillard, puede alcanzar óptimos resultados en términos de crecimiento y producción de organismos por unidad de tiempo, con la ventaja de aumentar su densidad en cultivo conforme transcurre el tiempo; esto garantiza la disponibilidad de la especie como presa viva en la larvicultura de peces.

Otro aspecto relevante en el manejo de los cladóceros es la calidad del agua en cultivo. El adecuado desarrollo y éxito en el cultivo está condicionado por factores ambientales que influyen en el crecimiento, la reproducción y la sobrevivencia; particularmente, la calidad y la disponibilidad del agua y del alimento en el medio de cultivo (Pérez et al., 2015; Tian et al., 2017; Ji et al., 2017). En cladóceros, se ha comprobado un efecto de la temperatura sobre el tiempo de desarrollo embrionario y post-embrionario, la fecundidad, la edad de la primípara, el tamaño corporal y la longevidad (Ismail et al., 2011; Huang et al., 2011; Viti et al., 2013). El incremento de la temperatura disminuye estos procesos debido a una alta tasa metabólica, donde las exigencias energéticas son mayores (Martínez-Jerónimo y Ventura-López, 2011; Chen et al., 2016 Dutta et al., 2017; Wei et al., 2018).

En la presente investigación durante el cultivo de $M$. spinosa, la temperatura osciló alrededor de $25,8^{\circ} \mathrm{C}$ en los dos tratamientos sin diferencia significativa; resultados cercanos a los reportados por diferentes autores para el cultivo de diferentes especies de cladóceros. Al respecto, Sipaúba-Tavares et al., (2014) señalan que Diaphanosoma birgei es más fecunda a temperaturas entre 25 a $30{ }^{\circ} \mathrm{C}$. Viti et al., (2013) reportan para Coronatella rectangula menor tiempo de desarrollo embrionario, alta fecundidad y menor número de estadios a temperaturas de $23,6 \pm 0,5^{\circ} \mathrm{C}$. Por otra parte, Huang et al., (2011) sugiere que Macrothrix rosea a temperaturas entre $20{ }^{\circ} \mathrm{C}$ y $25{ }^{\circ} \mathrm{C}$ se encuentran dentro del intervalo óptimo para el crecimiento y reproducción de esta especie. Ismail et al., (2011) observaron una disminución de la población del cladócero estuarino Daphniopsis australis, al incrementar la temperatura, y reportan temperatura ideal para la especie de $20{ }^{\circ} \mathrm{C}$. Por tanto, se puede considerar que la temperatura registrada en esta investigación se encontró dentro del rango normal registrado para diferentes especies de cladóceros, sin interferir en las variables de crecimiento y productivas de M. spinosa en cultivo.

El oxígeno disuelto es otro parámetro a considerar. Las concentraciones estables de este gas en el cultivo ayudan a mantener el metabolismo de los organismos, el aumento de la tasa de la ingesta de alimento y la estabilidad de los procesos aerobios reduciendo los compuestos amoniacales (Ferrão-Filho et al., 2003; Álvarez, 2010; Castilho et al., 2012; Prieto-Guevara 2013). Valores entre 3 a 8 mg/L son considerados óptimos en el cultivo ya que suple las necesidades de los organismos (Álvarez, 2010; Silva et al., 2014). En

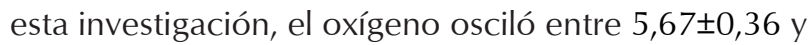
$5,74 \pm 0,56 \mathrm{mg} / \mathrm{L}^{\prime}$ sin diferencia significativa entre tratamientos. Por tanto, se infiere que este parámetro fue adecuado para el crecimiento poblacional de M. spinosa en cultivo.

En relación al pH, este es uno de los parámetros más importantes dentro del cultivo de cladóceros debido a que tiene un gran impacto en las tasas de sobrevivencia y la reproducción (Ghazy et al., 2011; Saha et al., 2017). Sobre el pH, Ghazy et al., (2011) consideran como rango óptimo de 7.9 a 8.3 para Daphnia magna. A su vez, Rottman et al., (2011) afirman que pH superior a 9,5 inhibe la producción de Moina sp., y aseguran que el rango de $\mathrm{pH}$ en cultivo debe ajustarse entre 
7 y 8 . Saha et al., (2017) reportan que Bosmina tripurae alcanza menor tiempo en su madurez sexual a $\mathrm{pH}$ 8.0, pero fecundidad y sobrevivencia más alta y el menor periodo embrionario en $\mathrm{pH}$ de 5 . De esta manera se puede inferir que el rango óptimo para el desarrollo de organismos zooplanctónicos es de 5 a 8, dependiendo de la especie en cultivo. En esta investigación, los va-

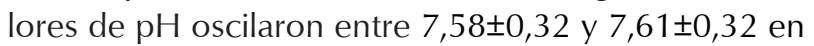
los dos tratamientos, por tanto, se puede inferir que los valores de $\mathrm{pH}$ registrados se encuentran en el rango indicado para el adecuado desarrollo de M. spinosa.

En general, la calidad del agua presentó condiciones óptimas, que no afectaron la dinámica poblacional de M. spinosa, y permitieron que ambos tratamientos presentarán características ambientales muy similares que favorecieron el análisis de los resultados, ya que se redujo la ocurrencia de variables interferentes.

Los resultados obtenidos en esta investigación, permiten concluir que el cladócero $M$. spinosa obtiene mayor densidad, tasa de crecimiento y rendimiento en cultivo y menor tiempo de duplicación cuando es alimentada con Chlorella sp. cultivada con medio F/2 de Guillard. Sin embargo, estudios sucesivos, donde se determine el valor nutricional de la dieta, se hacen necesarios para comprender la interacción de los organismos con la partícula alimenticia.

\section{Agradecimientos}

El Grupo de Investigación en Peces Nativos (GIPEN) adscrito a la Piscícola San Silvestre S.A, agradece a ISAGEN S.A. E.S.P por la financiación de la presente investigación en el marco del convenio 47-698, y al Instituto de Investigación Piscícola de la Universidad de Córdoba (CINPIC).

\section{Referencias}

Álvarez J. 2010. Caracterización limnológica de las ciénagas de Arcial, El Porro y Cintura (río San Jorge) y Bañó, Charco Pescao y Pantano Bonito (río Sinú), departamento de Córdoba. En: Rangel-Ch JO, Editor. Colombia Diversidad Biótica IX: Ciénagas de Córdoba: Biodiversidad-Ecología y Manejo Ambiental.

Azuraidi O, Yusoff F, Shamsudin M, Raha R, Alekseev V, Matias-Peralta $\mathrm{H}$. Effect of food density on male appearance and ephippia production in a tropical cladoceran, Moina micrura Kurz, 1874. Aquaculture. 2013;412-413:131-135.

Castilho M, Wisniewski C, Santos-Wisniewski M. Life cycle of Scapholeberis armata freyi Dumont \& Pensaert, 1983 (Cladocera, Daphnidae). Biota Neotrop. 2012;12(4):56-60.

Castro-Mejia J, Castro-Mejia G, Monroy-Dosta M, Davila-Sanchez F, Castro-Castellón A. Population density comparison of Cerio- dapnia dubia fed with bacteria obtained from Biofloc system. J Entomol Zool Stud. 2017; 5(5):2009-2012.

Cheban L, Grynko O, Dorosh I. Co-cultivation of Daphnia magna (Straus) and Desmodesmus armatus (chod.) Hegew. in recirculating aquaculture system wastewater. Arch Pol Fisheries. 2018;26(1):57-64.

Chen R, Tang H, Zhao F, Wu Y, Huang Y, Yang Z. Food availability and initial relative abundance determine the outcome of interspecific competition between two different-sized cladocerans. Int Rev Hydrobiol. 2016;101(1):105-112.

Cobos-Ruiz M, Paredes-Rodríguez J, Castro-Gómez J. Inducción de la producción de lípidos totales en microalgas sometidas a estrés nutritivo. Acta biol Colomb. 2016;21(1):17-26.

Day J, Gong Y, Hu Q. Microzooplanktonic grazers - A potentiaIly devastating threat to the commercial success of microalgal mass culture. Algal Res. 2017;27(1):356-365.

Dutta A, Kar S, Das P, Das U, Das S, Kar D. Studies on PhysicoChemical Aspects and Zooplankton Diversity of a Freshwater Wetland in Cachar, Assam. Int J Sci Environ Technol. 2017;6(3):1877- 1885.

Espinosa-Rodríguez C, Sarma S, Nandini S. Interactions between the rotifer Euchlanis dilatata and the cladocerans Alona glabra and Macrothrix triserialis in relation to diet type. Limnologica. 2012;42(1):50-55.

Ferrão-Filho A, Fileto C, Lopes N, Arcifa M. Effects of essential fatty acids and $\mathrm{N}$ and $\mathrm{P}$ limited algae on the growth of tropical cladocerans. Freshwater Biology. 2003;48(5):759-767. doi. org/10.1046/j.1365-2427.2003.01048.x

Fileto C, Arcifa M, Marchetti J, Turati I, Lopes N. Influence of biochemical, mineral and morphological features of natural food on tropical cladocerans. Aquat Ecol. 2007;41(4):557-568.

Fuentes-Reines J, Zoppi E, Morón E, Gámez D, López C. Conocimiento de la fauna de Cladocera (crustacea: branchiopoda) de la Ciénaga Grande de Santa Marta, Colombia. INVEMAR. 2012;41(1):121-164.

Gándara M, Leite R, Caraballo P. Historia de vida de Daphnia magna y Ceriodaphnia reticulata en condiciones de laboratorio: uso potencial como alimento para peces. Rev Colombiana Cienc. Anim. 2013;5(2):340-357.

Ghazy M, Habashy M, Mohammady Y. Effects of $\mathrm{pH}$ on survival, growth and reproduction rates of the crustacean, Daphnia magna. Aust J Basic \& Appl Sci. 2011;5(1):1-10.

González A. El Plancton de las aguas continentales. Secretaria general de la O.E.A. Serie de Biología; Monografía No. 33 Washington D.C. 1988.

Guillard RRL, Ryther JH. Studies of marine planktonic diatoms. I. Cyclotella nana Hustedt, and Detonula confervacea (Cleve) Gran. Can J Microbiol.1962;8(2):229-239.

Güntzel AM, Matsumura-Tundisi T, Rocha O. Life cycle of Macrothrix flabelligera Smirnov, 1992 (Cladocera, Macrothricidae), recently reported in the Neotropical region. Hydrobiologia. 2003;490(1):87-92. 
Guamán M, González N. 2016. Catálogo de microalgas y cianobacterias de agua dulce del Ecuador. Corporación Para La Investigación Energética. p.143.

Huang X, Shi X, Xu S, Liu G, Ma L, Sun Z. Life history characteristics of Macrothrix rosea (Jurine, 1820) (Cladocera, Macrothricidae) in laboratory conditions. J Limnol. 2011;70(2):248-254.

Ismail N, Qin G, Seuront L. Regulation of life history in the brackish cladoceran, Daphniopsis australis (Sergeev and Williams, 1985) by temperature and salinity. J Plankton Res. 2011;33(5):763777.

Ji G, Havens K, Beaver J, Fulton R. Response of Zooplankton to Climate Variability: Droughts Create a Perfect Storm for Cladocerans in Shallow Eutrophic Lakes. Water. 2017;9(764):1-20.

Kar S, Das P, Das U, Bimola M, Kar D, Aditya G. Culture of the zooplankton as fish food: observations on three freshwater species from Assam, India. Aquacult Aquarium Conserv Legis. 2017;10(5):1210-1220.

Khudyi O, Kushniryk O, Khuda L, Marchenko M. Differences in Nutritional Value and Amino Acid Composition of Moina macrocopa (Straus) Using Yeast Saccharomyces cerevisiae and Rhodotorula glutinis as Fodder Substrates. Int Lett Nat Sci. 2018;68:27-34.

Luna-Figeroa J, Arce E. Un menú diverso y nutritivo en la dieta de peces: "El alimento vivo". Agroproductividad. 2017;10(9):112116.

Marinho M, Lage O, Antunes S. Adecuacy of planctomycetes as supplementary food source for Daphnia magna. Antonie van Leeuwenhoek. 2017;111(6): 825-840.

Martínez-Jerónimo F, Ventura-López C. Population dynamics of the tropical cladoceran Ceriodaphnia rigaudi Richard, 1894 (Crustacea: Anomopoda). Effect of food type and temperature. J Environ Biol. 2011;32(1):513-521.

Mellisa S, Rahimi SAE, Umiati U. The effect of different live feeds on the growth and survival of comet goldfish Carrasius auratus larvae. In IOP Conference Series: Environ Earth Sci. 2018;216(1):12-25.

Muñoz M, Medina V, Cruz-Casallas P. Efecto del fotoperiodo y del alimento sobre la productividad de dos cladóceros nativos (Moina sp. y Diaphanosoma sp.) de la Orinoquia colombiana. Rev. U.D.C.A Actualidad \& Divulgación Científica, 2013;16(1):167-174.

Otero A, Muñoz M, Medina V, Cruz P. Efecto del alimento sobre variables productivas de dos especies de Cladóceros bajo condiciones de laboratorio. Rev MVZ Córdoba. 2013;18(1):36423647.

Pérez I, Villar A, Vargas M, Hernández-Vergara M, Pérez-Rostro C, Clemente A. Influencia de la temperatura y tipo de alimento en la historia de vida de Ceriodaphnia cornuta Sars (1885) (Crustacea: Cladocera). Revista Investigación y Ciencia- UAA. 2015;(64):11-18.
Prieto-Guevara M. 2013. Plancton regional y su potencial en acuicultura. Temas clave para la acuicultura. Centro de Investigaciones Piscícolas CIUC. Fondo editorial Universidad de Córdoba. ISBN 978-958-9244-61-6. Montería, Colombia. Primera edición. p.179.

Rottmann R, Graves J, Watson C, Yanong R. 2011. Culture Techniques of Moina: The Ideal Daphnia for Feeding Freshwater Fish Fry. IFAS CIR: 1054.

Saha H, Wisdom K, Devi A, Devi S, Kamei M, Biswas A, et al. Effects of Water pH on Life History Parameters of a New Bosminid Cladocera: Bosmina (Bosmina) Tripurae (Korinek, Saha and Bhattachaya, 1999) in Laboratory Condition. Bull Environ Contam Toxicol. 2017;99(1):23-26.

Silva E, Abreu C, Orlando T, Wisniewski C, Santos-Wisniewski M. Alona iheringula Sinev \& Kotov, 2004 (Crustacea, Anomopoda, Chydoridae, Aloninae): Life Cycle and DNA Barcode with Implications for the Taxonomy of the Aloninae Subfamily. PLoS ONE. 2014;9(5):1-7.

Silva-Benavides A. Evaluación de fertilizantes agrícolas en la productividad de la microalga Chlorella sorokiniana. Agron Mesoam. 2016;27(2):265-275.

Sipaúba-Tavares L, Truzzi B, Berchielli-Morais F. Growth and development time of subtropical Cladocera Diaphanosoma birgei Korinek, 1981 fed with different microalgal diets. Rev Braz J Biol. 2014;74(2):464-471.

Tian W, Zhang H, Zhang J, Zhao L, Miao M, Huang H. Responses of Zooplankton Community to Environmental Factors and Phytoplankton Biomass in Lake Nansihu, China. Pakistan J Zool. 2017;49(2):461-470.

Vásquez-Suárez A, Guevara M, González M, Cortez R, ArredondoVega B. Crecimiento y composición bioquímica de Thalassiosira pseudonana (Thalassiosirales: Thalassiosiraceae) bajo cultivo semi-continuo en diferentes medios y niveles de irradiancias. Rev Biol Trop. 2013;61(3):1003-1013.

Viti T, Wisniewski C, Orlando T, Santos-Wisniewski M. Life history, biomass and production of Coronatella rectangular (Branchiopoda, Anomopoda, Chydoridae) from Minas Gerais. Iheringia, Se Zoologia. 2013;103(2):110-117.

Wang Y, Xie N, Wang W. Effects of algal concentration and initial density on the population growth of Diaphanosoma celebensis Stingelin (Crustacea, Cladocera). Chin J Oceanol Limn. 2009;27(3):480-486.

Wei J, Zhao W, Wang S, Wang M, Wang X, Ji S, An H. Effect of temperature, salinity, and body length on the energy budget of Daphniopsis tibetana Sars (Cladocera: Daphniidae). Journal of Oceanology and Limnology (JOL). 2018;36(5):1812-1824.

Harold Oviedo: https://orcid.org/0000-0002-7783-1465 Edwin Herrera: https://orcid.org/0000-0002-5527-2375 Jenny Hoya: https://orcid.org/0000-0001-6507-9133 Martha Prieto: https://orcid.org/0000-0003-3458-4983 Ana Estrada: https://orcid.org/0000-0003-3585-3719 Jonny Yepes: https://orcid.org/0000-0001-6276-5488 